\title{
Perfil epidemiológico do câncer de colo do útero na Bahia (2015-2019)
}

\author{
Epidemiological profile of uterus cancer in Bahia (2015-2019)
}

Perfil epidemiológico del cáncer de útero en Bahia (2015-2019)

Anadir de Almeida Farias

ORCID: https://orcid.org/0000-0002-2124-531X Universidade Estadual do Sudoeste da Bahia, Brasil

E-mail: nnhfarias@gmail.com

Lyra Cândida Calhau Rebouças

ORCID: https://orcid.org/0000-0003-0576-4360

Universidade Estadual do Sudoeste da Bahia, Brasil

E-mail:lyracalhau@gmail.com

Ninalva de Andrade Santos

ORCID: https://orcid.org/0000-0001-7051-7230

Universidade Estadual do Sudoeste da Bahia, Brasi

E-mail: nsantos@uesb.edu.br

Cléber Souza de Jesus

ORCID: https://orcid.org/0000-0001-6727-3247

Universidade Estadual do Sudoeste da Bahia, Brasil E-mail: csjesus@uesb.edu.br

Marcela Rossi Ribeiro

ORCID: https://orcid.org/0000-0002-7874-6086

Universidade Estadual do Sudoeste da Bahia, Brasil

E-mail: marcelarossiribeiro@gmail.com

Gleide Magali Lemos Pinheiro

ORCID: https://orcid.org/0000-0002-0848-3348

Universidade Estadual do Sudoeste da Bahia, Brasil

E-mail: gleidemlp@uesb.edu.br

Charles Souza Santos

ORCID: https://orcid.org/0000-0001-5071-0359

Universidade Estadual do Sudoeste da Bahia, Brasil E-mail:charlesss@uesb.edu.br

Rebecca Calheira Barreto

ORCID: https://orcid.org/0000-0001-7555-6714

Universidade Estadual do Sudoeste da Bahia, Brasil

E-mail: rebeccacalheira@gmail.com

Maicla Oliveira da Silva

ORCID: https://orcid.org/0000-0002-4536-2262

Universidade Estadual do Sudoeste da Bahia, Brasil

E-mail: maicla64@gmail.com

Maycon Brandão dos Santos

ORCID: https://orcid.org/0000-0002-3012-267X

Universidade Federal do Recôncavo da Bahia, Brasil

E-mail: maycombrandaos@gmail.com

Evelin Matos Marambaia Souza

ORCID: https://orcid.org/0000-0001-9352-5524

Universidade Estadual do Sudoeste da Bahia, Brasil

E-mail: evelinmarambaia@yahoo.com.br

Esther Aderno Souza

ORCID: https://orcid.org/0000-0003-4565-9100

Universidade Estadual do Sudoeste da Bahia, Brasil

E-mail: estheraderno16@hotmail.com

Angelle Matias Melo

ORCID: https://orcid.org/0000-0002-8734-0563

Universidade Estadual do Sudoeste da Bahia, Brasil

E-mail: angellematias23@gmail.com

Micaela Leão de Sousa

ORCID: https://orcid.org/0000-0002-4726-5837

Universidade Estadual do Sudoeste da Bahia, Brasil

E-mail: micaelaleao_s@ @otmail.com

Letícia Souza Leal

ORCID: https://orcid.org/0000-0002-3290-0324

Universidade Estadual do Sudoeste da Bahia, Brasil

E-mail: leticiasouzaleal_@hotmail.com 


\begin{abstract}
Resumo
Objetivo: caracterizar o perfil epidemiológico do câncer do colo de útero na Bahia entre os anos de 2015 a 2019. Metodologia: trata-se de estudo de abordagem quantitativa, descritivo dos casos com resultados de citologias positivas para câncer de colo do útero, identificados a partir de exames citopatológicos, registrados no Sistema de Informação do Câncer e disponíveis no Departamento de Informática do Sistema Único de Saúde, entre os anos de 2015 a 2019. Resultados: os anos com menor e maior número de realização de exames citopatológicos foram 2015 e 2019 , respectivamente. Encontrou-se a cobertura de rastreamento de 32,0\% para os anos de 2017 a 2019. O quantitativo de exames alterados apresentou aumento discreto no período analisado. Quanto à realização prévia da citologia foi identificado que $81 \%$ das mulheres já haviam realizado anteriormente, $11 \%$ realizaram o exame pela primeira vez, $7 \%$ com essa informação ignorada. Prevaleceram os intervalos de realização do exame entre 1 ano e 2 anos. O rastreamento foi o principal motivo de realização do exame. A variável escolaridade é demasiadamente subnotificada. O carcinoma epidermóide invasor foi o mais frequente $(68,30 \%)$, seguido do adenocarcinoma In Situ (16,49\%) e adenocarcinoma invasor $(15,21 \%)$. A faixa etária mais acometida foi a de 35-44 anos $(31,1 \%)$. Conclusão: o alto número de resultados alterados suscita ser emergente implementação de políticas proativas de modo que a prevenção e o diagnóstico precoce do câncer de colo uterino seja minimizado de forma expressiva. Estudos com esse delineamento constituem importantes ferramentas de identificação da real situação epidemiológica oportunizando melhor tomada de decisão.
\end{abstract}

Palavras-chave: Câncer de colo do útero; Citologia; Epidemiologia.

\begin{abstract}
Objective: to characterize the epidemiological profile of cervical cancer in Bahia between the years 2015 to 2019. Methodology: this is a study with a quantitative, descriptive approach of cases with positive cytology results for cervical cancer, identified from Pap smear tests, registered in the Cancer Information System and available at the Informatics Department of the Unified Health System, between the years 2015 to 2019. Results: the years with the lowest and highest number of Pap smear tests were 2015 and 2019, respectively. A tracking coverage of 32.0\% was found for the years 2017 to 2019. The number of altered exams showed a slight increase in the period analyzed. As for the previous performance of cytology, it was identified that $81 \%$ of women had already performed it before, $11 \%$ underwent the exam for the first time, $7 \%$ with this information ignored. Intervals between 1 year and 2 years for the examination prevailed. Tracking was the main reason for performing the exam. The education variable is greatly underreported. Invasive squamous cell carcinoma was the most frequent (68.30\%), followed by In Situ adenocarcinoma (16.49\%) and invasive adenocarcinoma $(15.21 \%)$. The most affected age group was $35-44$ years $(31.1 \%)$. Conclusion: the high number of altered results suggests that the implementation of proactive policies is emerging so that the prevention and early diagnosis of cervical cancer is significantly minimized. Studies with this design are important tools for identifying the real epidemiological situation, providing opportunities for better decision-making.
\end{abstract}

Keywords: Cervical cancer; Cytology; Epidemiology.

\title{
Resumen
}

Objetivo: caracterizar el perfil epidemiológico del cáncer cervicouterino en Bahía entre los años 2015 a 2019. Metodología: se trata de un estudio con abordaje cuantitativo, descriptivo de casos con citología positiva para cáncer cervicouterino, identificados a partir de Papanicolaou, registrados en el Sistema de Información del Cáncer y disponibles en el Departamento de Informática del Sistema Único de Salud, entre los años 2015 a 2019. Resultados: los años con menor y mayor número de pruebas de Papanicolaou fueron 2015 y 2019, respectivamente. Se encontró una cobertura de seguimiento de $32.0 \%$ para los años 2017 a 2019. El número de exámenes alterados mostró un leve aumento en el período analizado. En cuanto a la realización previa de citología, se identificó que el $81 \%$ de las mujeres ya la había realizado antes, el $11 \%$ se sometió al examen por primera vez, el $7 \%$ con esta información ignorada. Prevalecieron intervalos entre 1 año y 2 años para el examen. El seguimiento fue la principal razón para realizar el examen. La variable educación no se informa en gran medida. El carcinoma epidermoide invasivo fue el más frecuente $(68,30 \%)$, seguido del adenocarcinoma in situ $(16,49 \%)$ y el adenocarcinoma invasivo $(15,21 \%)$. El grupo de edad más afectado fue el de 35 a 44 años (31,1\%). Conclusión: el elevado número de resultados alterados sugiere que está emergiendo la implementación de políticas proactivas para que la prevención y el diagnóstico precoz del cáncer cervicouterino se minimice significativamente. Los estudios con este diseño son herramientas importantes para identificar la situación epidemiológica real, brindando oportunidades para una mejor toma de decisiones.

Palabras clave: cáncer de cuello uterino; Citología; Epidemiología.

\section{Introdução}

O câncer de colo de útero (CCU) ou câncer cervical, é um problema de saúde pública global que acomete um contingente significativo de mulheres (Arbyn et al., 2020). De acordo com o Instituto Nacional de Câncer - INCA (2021a), o CCU está entre os mais incidentes, é o terceiro depois do câncer de pele não melanoma, e com taxas de mortalidade mais elevadas entre as mulheres no Brasil. 
Trata-se de uma patologia que possui como principal fator para sua gênese as infecções persistentes do Papilomavírus Humano - HPV, sendo os subtipos de HPV-16 e HPV-18 de alto risco, responsáveis pela maior parte dos casos. A fisiopatologia envolve alterações do processo de maturação do epitélio, predominantes das células escamosas, com diferentes graus de gravidade que dependem da proporção de células imaturas atípicas e espessura do epitélio envolvido (INCA, 2021b; Rezende, 2014).

Comumente, é uma doença de desenvolvimento lento, assintomática na fase inicial, mas que pode ser detectada por meio do rastreamento (INCA, 2021b). Atualmente, o exame citopatológico ou Papanicolau tem se mostrado como uma importante ferramenta no rastreamento do $\mathrm{CCU}$, e por conseguinte, na detecção e tratamento precoce. $\mathrm{O}$ exame tem como população alvo mulheres com idade entre 25 e 64 anos, que possuam colo do útero e que já tenham vivenciado, em algum momento da vida, relação sexual (Brasil, 2016).

Os altos índices de mortalidade por este tipo de câncer estão relacionados, principalmente, com baixo poder aquisitivo, dificuldade de acesso aos serviços de diagnóstico precoce e/ou tratamento adequado imediato. Esta realidade resulta, entre outras coisas, da distância na localização das unidades de saúde, número reduzido de profissionais de saúde para realização do Papanicolau, seguimento inadequado dos casos com resultados positivos, horário de atendimento limitado, bem como, interferências relacionadas a cultura e as relações de gênero (OPAS, 2016).

O CCU apesar de constituir uma problemática mundial têm apresentado número de casos com variações a depender das ações de prevenção implementadas em cada localidade. Nota-se, redução das taxas de incidência na maioria dos países desenvolvidos e tendência de crescimento ou manutenção dos casos nas nações em desenvolvimento. Porém, há de se considerar que, em quaisquer localidades, o CCU tem diagnóstico em fase invasiva mais incidente entre mulheres com baixo poder aquisitivo e residentes em áreas rurais, fato que ratifica a relação entre o agravo e vulnerabilidade socioeconômica (OPAS, 2016).

O CCU se caracteriza como uma problemática multifacetada fato que reitera a importância do mapeamento dos fatores associados a sua ocorrência a exemplo, o acometimento de mulheres jovens, que estão em plena vida produtiva e reprodutiva, ser uma doença que dispõe de tecnologia barata e de fácil realização para o diagnóstico e tratamento precoce, e ainda, porque, as mortes causadas por essa patologia são consideradas evitáveis, dada as incontestáveis evidências de que é um dos tipos de câncer de mais fácil prevenção e tratamento eficaz quando detectado precocemente (OPAS, 2016).

Diante do exposto, surgiu o seguinte questionamento: qual perfil epidemiológico do câncer do colo de útero na Bahia? O estudo da problemática se justifica em virtude da capacidade dos trabalhos epidemiológicos possibilitarem o conhecimento aprofundado da situação de saúde e a implementação de políticas públicas que possibilitem a prevenção do CCU e a melhoria da atenção à saúde da mulher, reduzindo de forma expressiva a incidência e a mortalidade do câncer de colo do útero. Nesse contexto, o principal objetivo deste estudo é caracterizar o perfil epidemiológico do câncer do colo de útero na Bahia, entre os anos de 2015 a 2019.

\section{Metodologia}

Estudo de natureza descritiva e de abordagem quantitativa dos casos positivos para câncer de colo do útero, identificados a partir de exames citopatológicos do colo do útero realizados no estado da Bahia entre os anos de 2015 a 2019.

O ano de 2020 não foi incluído no recorte temporal por não expressar um retrato fidedigno das políticas de saúde devido o cenário atípico ocasionado pela pandemia de Covid-19, com notável redução da oferta e acesso ao referido exame. Já o ano de 2021, não haviam informações disponíveis no sistema no período da coleta, que se deu entre julho e agosto de 2021.

O estudo foi realizado através da busca eletrônica das informações referentes aos resultados dos exames citopatológicos registrados no Sistema de Informação do Câncer (SISCAN) que é uma versão em plataforma Web do Departamento de 
Informática do Sistema Único de Saúde (DATASUS). Para a realização do estudo foram considerados os dados de exames citopatológicos do colo do útero realizados entre 2015 a 2019, em mulheres residentes da Bahia.

Os dados foram organizados para processamento por meio do Microsoft Office Excel® 2013 e apresentados através de tabelas e figuras para melhor assimilação e exposição. Os dados foram analisados quantitativamente e de maneira descritiva. Para análise, considerou-se as seguintes variáveis: faixa etária, escolaridade, histórico de realização do exame e o resultado do exame citopatológico que seguiu classificação do Sistema Bethesda de 2001 e a Nomenclatura Brasileira para Laudos Citopatológicos Cervicais definida pelo Instituto Nacional de Câncer José Alencar Gomes da Silva (INCA, 2012).

O estudo não foi submetido ao Comitê de Ética em Pesquisa (CEP), pois entende-se que os dados do DATASUS além de não identificarem os participantes, são de domínio público. Estando de acordo com a resolução de número 466 de 12 de dezembro de 2012 do Conselho Nacional de Saúde (CNS).

\section{Resultados e Discussão}

O levantamento dos dados disponibilizados pelo DATASUS evidenciou que entre 2015 a 2019 foram realizados na Bahia 2.421.217 exames citopatológicos do colo do útero, sendo que de 2015 a 2018 houve crescimento significativo e de 2018 a 2019, os dados se mantiveram quase que inalterados. Em 2015 foram realizados o menor quantitativo, 331.008 e em 2019 o maior número que foi 585.049 exames (Figura 1).

Figura 1 - Distribuição da realização de exames citopatológicos no estado da Bahia (2015 a 2019).

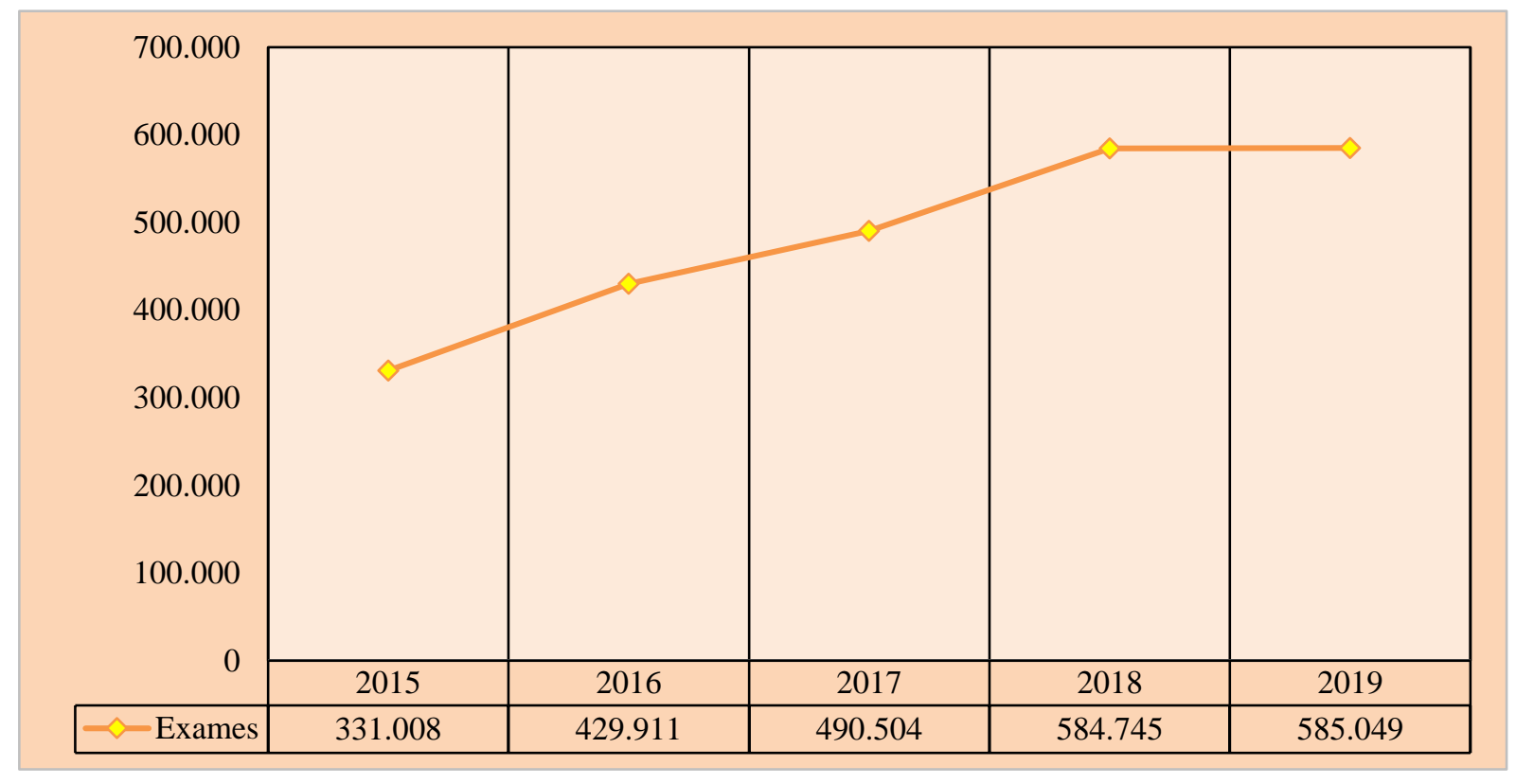

Fonte: DATASUS (2021).

Esse aumento na realização dos exames entre 2015 a 2018 e discreto de 2018 a 2019, pode justificar-se por diversas questões, como a criação e aprimoramento de programas de educação em saúde, busca ativa das mulheres para a realização do exame e investigação com respectiva intervenção sobre os fatores que influenciam na adesão à realização do exame de rastreamento do CCU (Ribeiro \& Andrade, 2016).

O exame citopatológico é o método amplamente utilizado para rastreamento do CCU, possui tecnologia simples, baixo custo e fácil realização, é considerado de alta eficácia para a detecção de lesões precursoras e câncer in situ (Brasil, 2016). Uma alta cobertura organizada da população alvo é ferramenta indispensável para a redução da incidência e da mortalidade por CCU. 
A OMS preconiza no mínimo a cobertura de $80 \%$ para redução da incidência de $60 \%$ a $90 \%$. O Brasil estabeleceu a meta de cobertura de $85 \%$ da realização de exames entre as mulheres da faixa etária preconizada até 2022 (WHO, 2002; Brasil, 2011).

Este estudo utilizou a projeção da população feminina alvo para o exame, ou seja, de 25 a 64 anos da Bahia no ano de 2019, 4.074.624 pessoas, e o quantitativo de citopatológicos total dos anos de 2017, 2018 e 2019, 1.309.604, para verificar a cobertura de exames citopatológicos (IBGE, 2021; DATASUS, 2021; INCA, 2014). Obteve-se assim a taxa de cobertura de 32\% para o estado da Bahia. Esse quantitativo reduzido pode justificar-se devido as limitações do próprio indicador e também aquelas relacionadas ao SISCAN como a alimentação deficiente do sistema e ausência dos dados provenientes da rede privada. Ainda, sofre influencias do grupo de mulheres que ainda não tem acesso ao serviço.

Ainda que a cobertura dos citopatológicos encontra-se abaixo do desejável, os dados obtidos por meio desta pesquisa evidenciam o aumento da realização do exame nos anos analisados. Nota-se crescimento expressivo entre os anos de 2015 a 2019 (Figura 1), fato que pode ser analisado sob a égide das melhorias ao acesso as ações e aos serviços de saúde que vem se desenrolando ao longo dos anos no Sistema Único de Saúde, não só no quesito da saúde da mulher, mais na saúde como um todo.

No que se refere aos exames com resultados alterados é possível notar que na medida em que aumentam a realização de exames citopatológicos, estes também aumentam, mesmo que de forma discreta (Figura 2).

Figura 2 - Exames realizados e exames alterados no estado da Bahia, segundo ano competência (2015 a 2019).

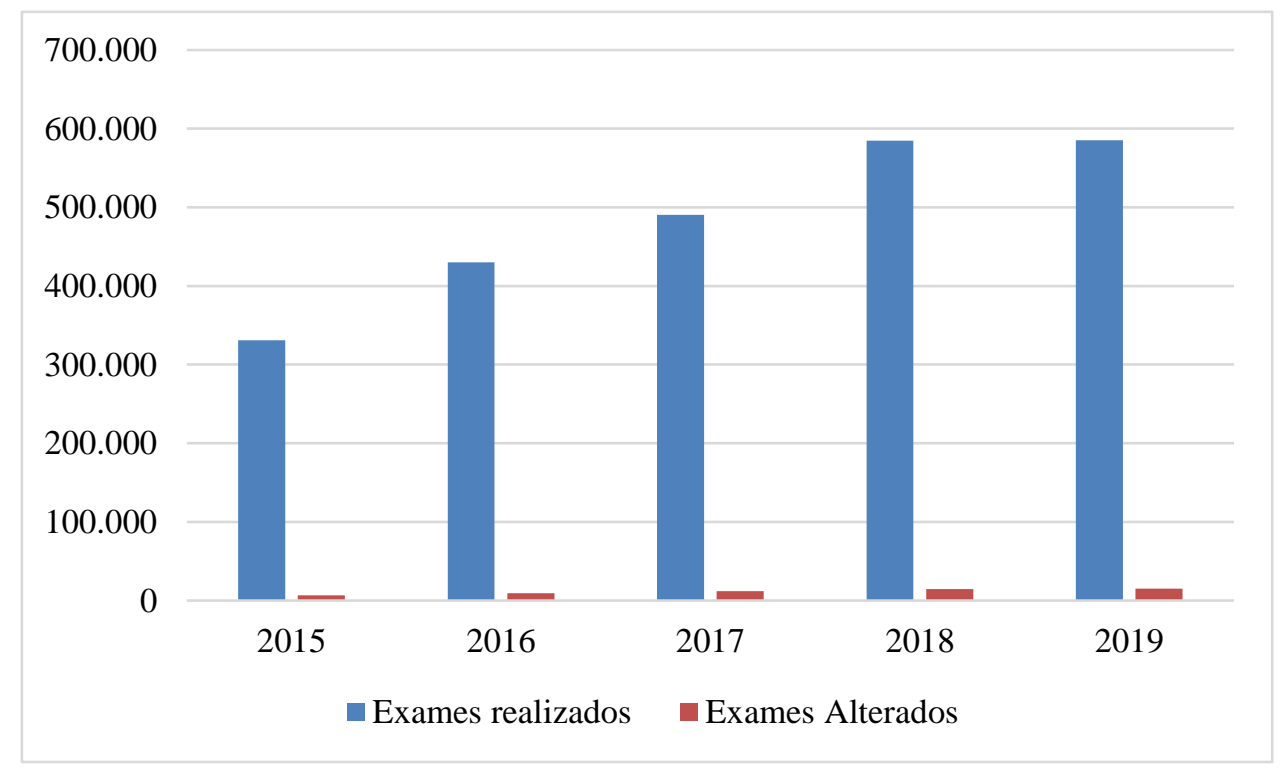

Fonte: DATASUS (2021).

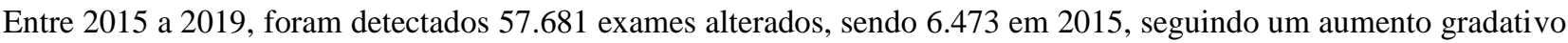
(Figura 2). Este aumento é esperado, seguindo a lógica de que ao aumentar a cobertura de exames, capta-se mais mulheres e assim, pode-se identificar mais o CCU e também as alterações que o precedem.

A variável exame alterado é relacionada a uma ou mais atipias e não englobam as alterações benignas, como inflamação e reparação. Podem ser classificadas em: células atípicas de significado indeterminado; células escamosas atípicas de significado indeterminado; células glandulares atípicas de significado indeterminado e células atípicas de origem indefinida, ou sejam alterações possivelmente não neoplásicas, mas que não se pode afastar a possibilidade da existência de lesões intraepiteliais de alto grau (INCA, 2012). 
Em células escamosas, as lesões intraepiteliais baixo grau compreende os danos provocados pelo efeito citopático viral do HPV as quais possuem grandes possibilidades de regressão espontânea. A lesões intraepiteliais alto grau possuem maiores probabilidades de progredirem para o câncer invasivo, são predominantementes ocasionadas pelos tipos de maiores probabilidades oncogênicas, estas devem ser tratadas o mais breve possível (INCA, 2012; Brasil, 2016). Os carcinomas epidermóides invasores também tem origem das células escamosas, representando cerca de 90\% de todos casos (INCA, 2021c).

No que diz respeito as alterações glandulares, os adenocarcinomas apresentam maiores possibilidades de erro no diagnóstico e consequente, evolução negativa, devido as lesões se desenvolverem a partir das células glandulares produtoras de muco do endocévice, as quais podem não serem identificadas no momento da leitura da lâmina. $\mathrm{O}$ adenocarcinoma in situ referese a fase inicial da patologia e o adenocarcinoma invasor, apesar de ser menos incidente, é mais agressivo (Diz \& Medeiros, 2009).

O fluxograma proposto pelo Ministério da Saúde orienta as condutas de seguimento diante dos resultados com alterações significativas nas unidades de atenção básica de acordo com o tipo de lesão, no geral predominam o encaminhamento para colposcopia ou repetição da citologia (Brasil, 2016). Fazer exame de rastreamento sem tratamento adequado das alterações não é eficiente, logo, não é o bastante para assegurar a redução da morbidade e da mortalidade por CCU (Laganá, 2013). A relevância do rastreamento organizado e do seguimento adequado para uma análise de forma ampliada e detalhada e o tratamento eficiente das pessoas com exames alterados é evidenciada em estudos como, Santos et al., (2019) e Farias, Barbieri (2016).

A Figura 3 expressa informações sobre a realização prévia da citologia do colo, $81 \%$ das mulheres informaram já terem feito o exame anteriormente, $11 \%$ sem realização anterior do exame, ou seja, apesar de estarem na faixa etária alvo, realizavam o procedimento pela primeira vez. Ademais, chama atenção o fato de 7\% das mulheres não saberem se já haviam realizado o exame anteriormente ou não.

Figura 3 - Informação referente a realização anteriormente do exame citopatológico do colo do útero no estado da Bahia (2015 a 2019).

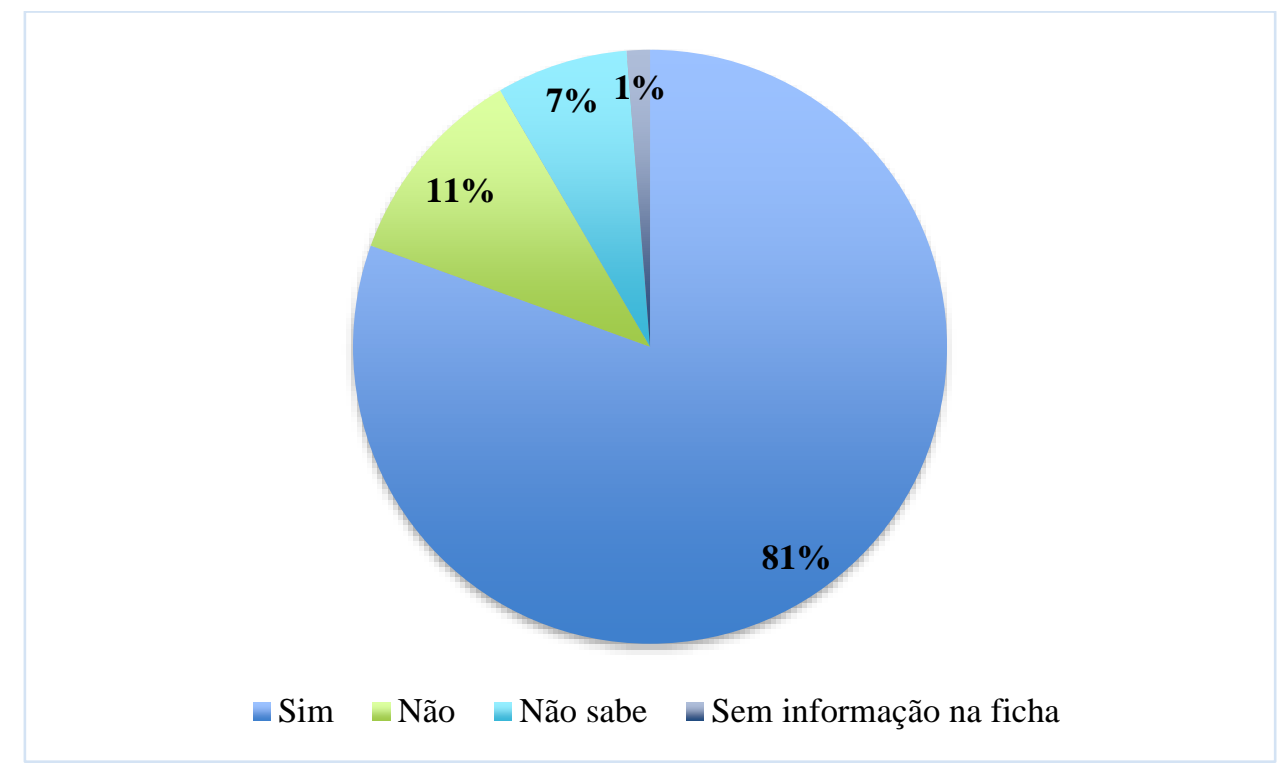

Fonte: DATASUS (2021).

Um olhar sobre os $81 \%$ que representa aquelas que já realizaram o exame anteriormente, ou seja, a maioria da amostra analisada, pode ter como explicação o fato de haver conscientização acerca da importância do exame. Inquieta-nos a qualidade 
do procedimento realizado (desde a coleta até a leitura das lâminas) e, sobretudo, o adequado seguimento dos casos positivos em tempo hábil.

Um estudo obteve resultado semelhante ao avaliar o conhecimento das mulheres quanto as ações de prevenção a saúde, foram identificados que 54\% apresentaram consciência quanto a relevância do exame e a realização do mesmo com intervalo anual (Miranda et al., 2018). Todavia, é preciso considerar as mulheres que associam a realização do exame para detecção de alterações benignas, a exemplo, processo inflamatório.

No Brasil, a recomendação é que se inicie a coleta do exame em mulheres aos 25 anos, que já iniciaram a vida sexual, pois existe o consenso de que antes disso ainda não se entrou em contato com o principal preditor da doença, o vírus HPV. Além disso, abaixo dessa faixa etária a incidência do câncer invasor do colo do útero é baixa e por se tratar de um problema que desenvolve lentamente, o rastreamento é menos eficiente para detectá-lo nessa situação (Brasil, 2016).

No entanto, algumas questões podem antecipar ou distanciar essa ação, como: sentimentos de vergonha, questões culturais e medo; inadequações do serviço de saúde; falta de compreensão das orientações médicas e a desinformação sobre o exame citopatológico do colo do útero (Nascimento \& Araújo, 2014).

A falta de informação supracitada pode estar relacionada com o dado da Figura 3, na qual 7\% das mulheres informaram que não sabem se realizou citologia anterior. Acreditamos que essa realidade pode ser explicada pela carência de informação por parte dos profissionais de saúde ou pela baixa escolaridade que dificulta o entendimento da orientação disponibilizada.

A Figura 4 mostra o tempo entre o último exame citopatólogico e o atual no decorrer dos anos de 2015 a 2019. No geral, nota-se aumento em todos os seguimentos durante os 5 anos, com ênfase para realização com 1 ano, seguido de 2 anos, 3 anos, menos de um ano, e 4 anos ou mais.

Figura 4 - Tempo entre o último exame citopatólogico e o atual no estado da Bahia, segundo ano de competência (2015 a 2019).

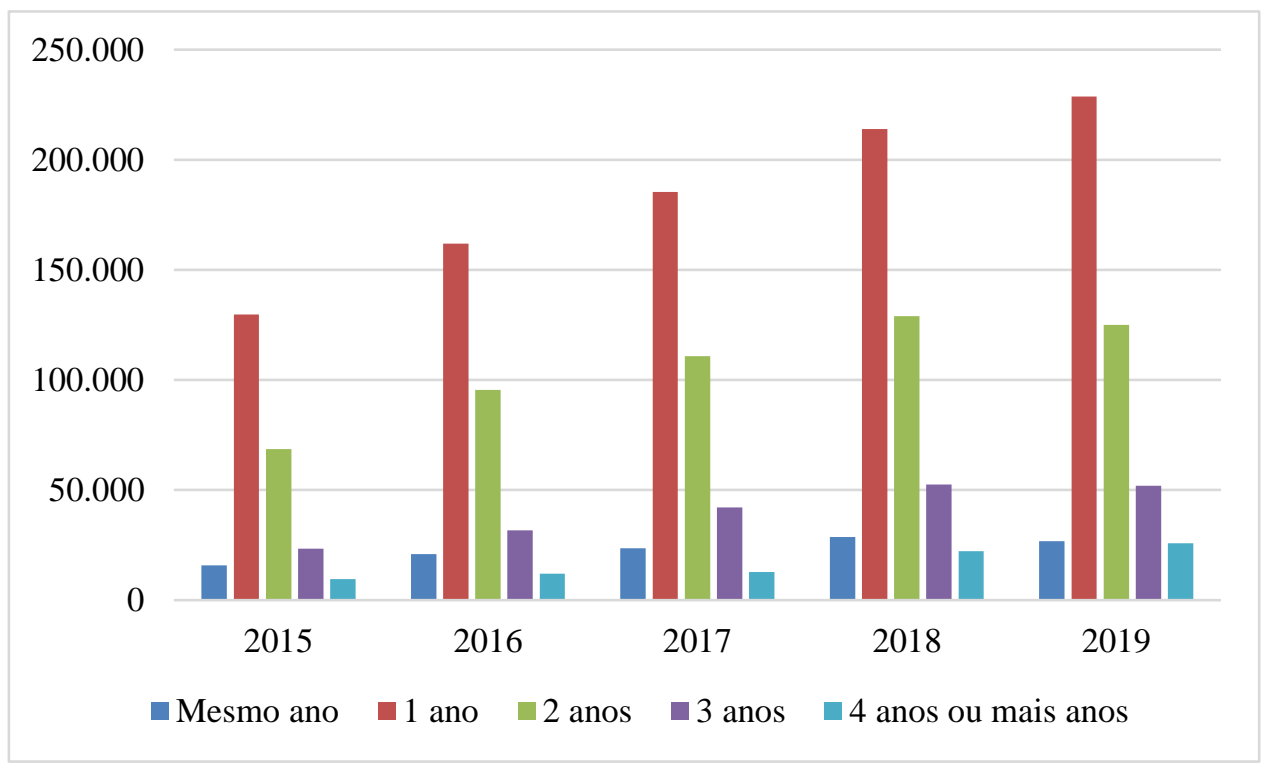

Fonte: DATASUS (2021).

No que se refere a periodicidade da realização do exame citopatológico, devido ao longo período de desenvolvimento das lesões precursoras e do CCU, é recomendada a realização de dois exames com intervalo anual, se ambos apresentarem resultados negativos, o próximo será realizado, com intervalo de três anos (Brasil, 2016).

O estudo exploratório realizado em São Paulo chama atenção para o fato de o Brasil não realizar triagem adequada do câncer de colo uterino, de forma organizada. Prevalece a realidade da realização do exame de forma oportunística, ou seja, 
quando a mulher ocasionalmente vai a unidade de saúde sem atender o tempo entre realização de um e outro exame (Souza et al., 2013). Quando o exame é realizado fora do intervalo de tempo recomendado, resulta em atendimento da meta proposta na localidade, mas, por outro lado, sem o devido impacto na minimização do número de casos de CCU. A realidade da Bahia corrobora com o estudo supracitado, ainda que a Figura 4 evidencie uma predominância na realização com 1 ano, a maioria das mulheres realiza o exame fora da periodicidade recomendada.

No que diz respeito aos motivos de realização do citopatológico, o rastreamento ganha destaque em todos os anos, seguido dos exames de seguimento, que são destinados para acompanhamento ou pós tratamento de lesão, e os de repetição que são indicados quando um primeiro exame tem o resultado alterado por células escamosas atípicas de significado incerto ou por lesão de baixo grau (Tabela 1).

Tabela 1 - Motivos da realização do citopatológico no estado da Bahia, segundo ano de competência (2015 a 2019).

\begin{tabular}{ccccccc}
\hline Ano competência & Rastreamento & \% & Repetição & \% & Seguimento & \% \\
\hline $\mathbf{2 0 1 5}$ & 318.978 & 13,50 & 366 & 7,28 & 11.664 & 21,88 \\
\hline $\mathbf{2 0 1 6}$ & 417.899 & 17,69 & 608 & 12,10 & 11.404 & 21,40 \\
\hline $\mathbf{2 0 1 7}$ & 476.140 & 20,15 & 1.303 & 25,93 & 13.061 & 24,51 \\
\hline $\mathbf{2 0 1 8}$ & 574.499 & 24,31 & 1.350 & 26,87 & 8.896 & 16,69 \\
\hline $\mathbf{2 0 1 9}$ & 575.379 & 24,35 & 1.398 & 27,82 & 8.272 & 15,52 \\
\hline
\end{tabular}

*Repetição - Exame Alterado ASCUS/Baixo Grau. Fonte: DATASUS (2021).

Reafirmando, uma análise do período de 2014 e 2015 constatou que em todas as regiões do país, o motivo mais frequente foi o rastreamento $(96,6 \%)$ (INCA, 2017).

No que tange a escolaridade, a tabela 2 demostra o quão subnotificada é esta variável no estado da Bahia. Essa realidade permeou todo o período analisado fato que suscita a necessidade de capacitação dos profissionais de saúde no campo da epidemiologia.

Tabela 2 - Escolaridade de mulheres que realizaram exame citopatológico no estado da Bahia, segundo os ano de competência (2015 a 2019).

\begin{tabular}{cccccc}
\hline Escolaridade & $\mathbf{2 0 1 5}$ & $\mathbf{2 0 1 6}$ & $\mathbf{2 0 1 7}$ & $\mathbf{2 0 1 8}$ & $\mathbf{2 0 1 9}$ \\
\hline Analfabeto(a) & 112 & 0 & 0 & 0 & 6 \\
\hline Ensino Fundamental Incompleto & 397 & 0 & 0 & 0 & 7 \\
\hline Ensino Fundamental Completo & 311 & 1 & 0 & 0 & 3 \\
\hline Ensino Médio Completo & 295 & 0 & 0 & 0 & 6 \\
\hline Ensino Superior Completo & 38 & 0 & 0 & 0 & 2 \\
\hline Ignorado & 329.855 & 429.910 & 490.504 & 584.745 & 585.025 \\
\hline Total & 331.008 & 429.911 & 490.504 & 584.745 & 585.049 \\
\hline
\end{tabular}

Fonte: DATASUS (2021).

Esse achado guarda relação com o estudo que identificou que 7 das macrorregiões do estado do Maranhão deixaram de caracterizar a variável escolaridade no momento de realização do exame citopatológico, em mais de $70 \%$ das vezes em que as mulheres foram realizar o exame (Nogueira et al., 2021). Essa deficiência no preenchimento de fichas e consequentemente, na alimentação do sistema pode mascarar a correlação entre o agravo e questões socioeconômicas.

Essa inferência encontra respaldo em estudo que verificou que a baixa escolaridade e a raça/cor não branca foram os principais fatores determinantes para a ocorrência de alterações citológicas de alto grau no colo de útero das mulheres estudadas 
(Melo et al., 2017). Neste estudo, a variável raça/cor não pôde ser analisada, por não está disponível no DATASUS para o estado da Bahia.

A Figura 5 demostra os exames com diagnóstico para CCU segundo a faixa etária. Estes, totalizam 388 casos entre os anos de 2015 a 2019, sendo 265 casos de carcinoma epidermóide invasor, adenocarcinoma in situ 64 e adenocarcinoma invasor 59.

Figura 5 - Exames com diagnóstico de carcinoma epidermóide invasor, adenocarcinoma in situ e adenocarcinoma invasor segundo faixa etária no estado da Bahia (2015 a 2019).

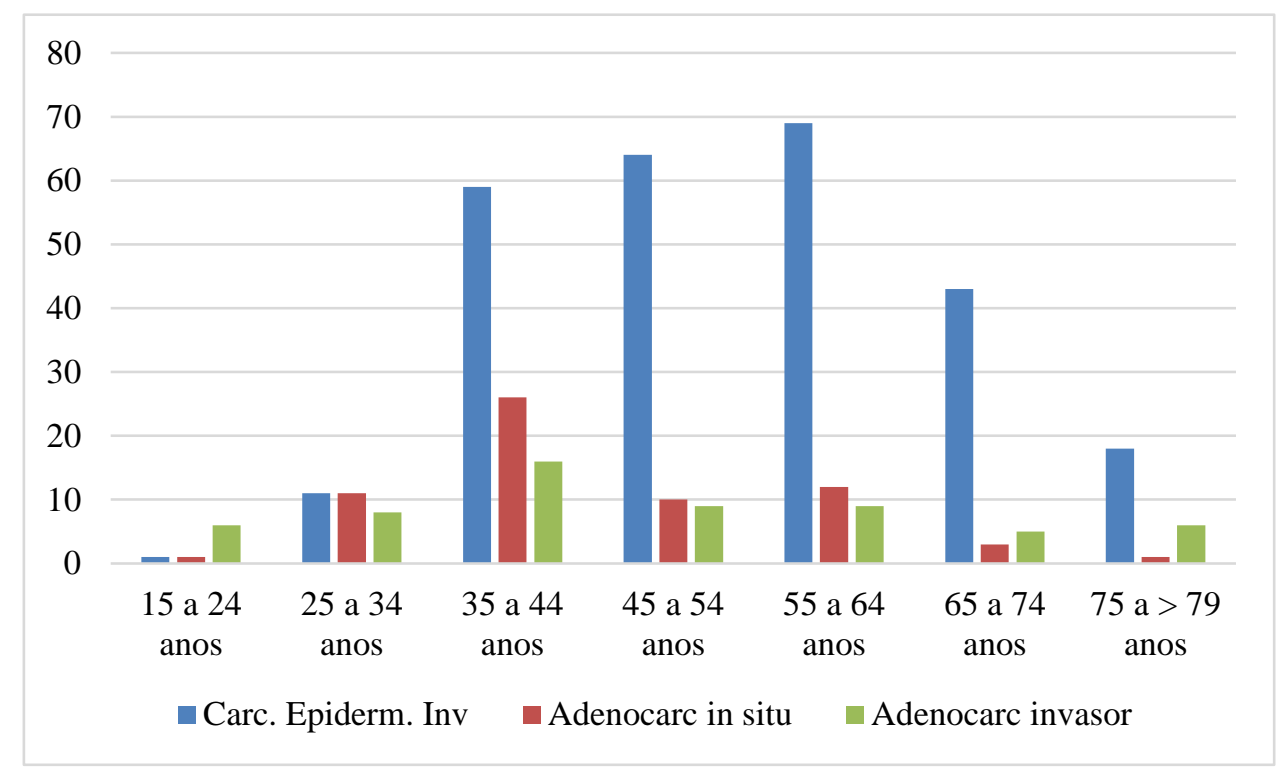

Fonte: DATASUS (2021).

Quanto à faixa etária é possível identificar quatro grupos com maior incidência de CCU dentro do intervalo de tempo do estudo. São eles, mulheres entre 35-44 anos (31,08\%); 45-54 anos (25,54\%); 55-64 anos (27,69\%); 65-74anos (15,69\%). Em ambos os grupos o carcinoma epidermóide invasor mais frequente (Figura 5), validando o exposto pelo INCA (2021c) que aponta esse tipo como mais incidente no país, fato que pode ser explicado pelo diagnóstico tardio.

No geral, o câncer cervical acomete mulheres na faixa etária reprodutiva, concentrando-se naquelas com idade a partir de 35 anos, tendo a faixa etária em que ocorre o pico da incidência de 45 a 50 anos (INCA, 2021c). Os dados deste estudo guardam relação com esta cifra nacional.

Todavia, a incidência do CCU vem diminuindo, nas últimas décadas, de forma mais evidente em países que passam por processo de transição socioeconômica (Globocan, 2012).

A exemplo disso, o Brasil possui uma taxa de incidência estimada que expressa valores intermediários em relação aos países em desenvolvimento, e elevadas quando comparadas às de países desenvolvidos com programas de detecção precoce bem estruturados, como os países europeus, Japão, Estados Unidos e Canadá. Na análise regional do país, a região a qual pertence o estado do estudo, o Nordeste, ocupa a segunda colocação de mais incidente (16,10/100 mil), a região sudeste se encontra na quinta posição (12,01/100 mil) (INCA, 2019).

\section{Conclusão}

$\mathrm{O}$ estudo do perfil epidemiológico constitui uma relevante ferramenta que viabiliza o conhecimento dos fatores associados ao agravo em uma determinada localidade e subsidia as ações no campo da saúde. Neste sentido, prevalece o olhar 
que repercussões referentes a esse tipo de câncer estão atreladas a questões sociais, biológicas, econômicas, políticas e ambientais. Assim, oportuna identificação desses aspectos propicia meios para intervenções no que diz respeito, ao controle da problemática.

Consideramos positivo o crescente número de realização da citologia no decorrer do período analisado. Esse aumento pode ser justificado pelas melhorias na atenção à saúde da mulher. A identificação de cobertura de $32 \%$ (muito abaixo do recomendado pela OMS) no período estudado é preocupante. Cobertura baixa e/ou espontânea (desorganizada) são comumente associadas a maior incidência de CCU.

O CCU demostrou predominância no estado da Bahia em mulheres que se enquadram na faixa etária de 35-44 anos, seguindo a cifra nacional, sendo o carcinoma epidermóide invasor mais frequente fato que pode ser indicativo de má qualidade nas ações de rastreamento e/ou seguimento das lesões precursoras. A ausência da variável raça/cor e a subnotificação do quesito escolaridade sinalizam a desvalorização da epidemiologia descritiva para a análise da problemática e, sobretudo, tomada de decisão acerca dos fatores associados a alta incidência de CCU em nosso país.

Apesar dos esforços ainda há muito o que ser feito no que diz respeito ao controle do câncer de colo do útero na Bahia, tanto em relação a educação em saúde quanto aprimoramento da implantação do SISCAN e capacitação profissional.

O estudo possuiu como limitação a utilização de dados de fonte secundária, cuja alimentação deficiente do sistema obscurece o mapeamento da realidade em sua plenitude. Uma outra fragilidade é o fato da análise ter subsídios do SISCAN e este abranger todo o serviço público e privado apenas de forma complementar, deixando lacuna de informação, o que solidifica a importância de o câncer de colo útero ser incluído na lista de agravos de notificação obrigatória facilitando o monitoramento da rede privada. Ainda assim, este estudo constitui-se como um importante parâmetro para o desenvolvimento de políticas públicas, possibilitando abordagens preventivas mais eficientes por parte dos gestores da saúde pública.

\section{Referências}

Arbyn, M., Weiderpass, E., Bruni, L., Sanjosé, S., Saraiya, M., Ferlay, J. \& Bray, F. (2020). Estimates of incidence and mortality of cervical cancer in 2018: a worldwide analysis. Lancet Glob Health. 8(2). 10.1016 / S2214-109X (19) 30482-6.

Brasil (2016). Instituto Nacional de Câncer José Alencar Gomes da Silva. Coordenação de Prevenção e Vigilância. Divisão de Detecção Precoce e Apoio à Organização de Rede. Diretrizes brasileiras para o rastreamento do câncer do colo do útero. https://www.inca.gov.br/sites/ufu.sti.inca.local/files//media/document//diretrizesparaorastreamentodocancerdocolodoutero_2016_corrigido.pdf.

Brasil (2011). Ministério da Saúde. Plano de ações estratégicas para o enfrentamento das doenças crônicas não transmissíveis (DCNT) no Brasil, $2011-2022$. Brasília: Ministério da Saúde. https://bvsms.saude.gov.br/bvs/publicacoes/plano_acoes_enfrent_dcnt_2011.pdf.

Departamento de Informática do Sistema Único de Saúde (DATASUS) (2021). Sistema de Informação do Câncer - SISCAN. Distribuição de exames citopatológicos por local de residência no estado da Bahia em 2014. < http://tabnet.datasus.gov.br/cgi/dhdat.exe?SISCAN/cito_colo_residba.def>.

Diz, M. D. P. E, \& Medeiros, R. B. (2009). Câncer de colo uterino - fatores de risco, prevenção, diagnóstico e tratamento. Rev Med (São Paulo). 88(1):7-15. 10.11606/issn.1679-9836.v88i1p7-15.

Farias, A. C. B. \& Barbieri, A. R. (2016). Seguimento do câncer de colo de útero: Estudo da continuidade da assistência à paciente em uma região de saúde. Escola Anna Nery. 20 (4). 10.5935/1414-8145.20160096.

Globocan (2012). Global Cancer Observatory. http://globocan.iarc.fr/Default.aspx.

Instituto Brasileiro de Geografia e Estatística (IBGE) (2021). Estimativa populacional. https://www.ibge.gov.br/estatisticas/sociais/populacao/9103-estimativasde-populacao.html?edicao $=17283 \& \mathrm{t}=$ downloads.

Instituto Nacional de Câncer José Alencar Gomes da Silva (2021b) (INCA). Câncer do colo do útero. https://www.inca.gov.br/tipos-de-cancer/cancer-do-colodo-utero.

Instituto Nacional de Câncer José Alencar Gomes da Silva (2021c) (INCA). Conceito e Magnitude. https://www.inca.gov.br/controle-do-cancer-do-colo-doutero/conceito-e-magnitude.

Instituto Nacional de Câncer José Alencar Gomes da Silva (2012) (INCA). Coordenação-Geral de Prevenção e Vigilância. Divisão de Detecção Precoce e Apoio à Organização de Rede. Nomenclatura brasileira para laudos citopatológicos cervicais. (3a ed.), https://www.inca.gov.br/sites/ufu.sti.inca.local/files/media/document/nomenclatura-brasileira-para-laudos-citopatologicos-cervicais-2012.pdf. 
Research, Society and Development, v. 11, n. 1, e41911125077, 2022

(CC BY 4.0) | ISSN 2525-3409 | DOI: http://dx.doi.org/10.33448/rsd-v11i1.25077

Instituto Nacional de Câncer José Alencar Gomes da Silva (2019) (INCA). Estimativa 2020: incidência do Câncer no Brasil. Rio de Janeiro: INCA. D<https://www.inca.gov.br/sites/ufu.sti.inca.local/files//media/document//estimativa-2020-incidencia-de-cancer-no-brasil.pdf>.

Instituto Nacional do Câncer José Alencar Gomes da Silva (INCA) (2014). Ficha técnica de indicadores das ações de controle do câncer do colo do útero. https://www.inca.gov.br/sites/ufu.sti.inca.local/files/media/document/fichatecnicaindicadorescolo14.pdf.

Instituto Nacional de Câncer José Alencar Gomes da Silva (2017) (INCA). Monitoramento das ações de controle dos cânceres do colo do útero e de mama. Informativo Deteção Precoce, 8 (1). Disponível em: https://www.inca.gov.br/sites/ufu.sti.inca.local/files//media/document//informativo-deteccaoprecoce-12017.pdf

Laganá, M. T. C., Silva, M. M. P. S., Lima, L. F. \& França, T. L. B. (2013). Alterações citopatológicas, doenças sexualmente transmissíveis e periodicidade dos exames de rastreamento em Unidade Básica de Saúde. Rev Bras Cancerol. 59 (4). 10.32635/2176-9745.RBC.2004v59n1.969.

Melo, W. A., Pelloso, S. M., Alvarenga, A, \& Carvalho, M. D. B. (2017). Fatores associados a alterações do exame citopatológico cérvico-uterino no Sul do Brasil. Rev. Bras. Saúde Matern. Infant., Recife. 17 (4). 10.1590/1806-93042017000400002.

Miranda, A. P., Rezende, E. V. \& Romero, N. S. A. (2018). Percepção e adesão das mulheres quanto ao exame citopatológico. Revista Nursing, 21 (246): 2435 2438. http://www.revistanursing.com.br/revistas/246/pg29.pdf.

Nascimento, R. G. \& Araújo, A. (2014). Falta de periodicidade na realização do exame citopatológico do colo uterino: motivações das mulheres. Rev Min Enferm. 18 (3). 10.5935/1415-2762.20140041.

Nogueira, L. M., Costa, S. S., Costa, I. S., Lopes, D. L., Aguiar, D. C. C. \& Silva, L. C. M. (2021). Caracterização epidemiológica do câncer do colo uterino, anterior à implantação do calendário vacinal para o HPV no estado do Maranhão. Revista Eletrônica Acervo Saúde, 13 (2). doi:10.25248/reas.e5804.2021

Organização Pan-Americana da Saúde (2016) (OPAS). Controle integral do câncer do colo do útero. Guia de práticas essenciais. Washington, DC. https://iris.paho.org/bitstream/handle/10665.2/31403/9789275718797-por.pdf?sequence=1\&isAllowed=y.

Rezende (2014). Obstetrícia fundamental/Carlos Antonio Barbosa Montenegro, Jorge de Rezende Filho. (13a ed.), Guanabara Koogan.

Ribeiro, J. C. \& Andrade, S. R. D. (2016). Vigilância em saúde e a cobertura de exame citopatológico do colo do útero: revisão integrativa. Texto \& ContextoEnfermagem. 25 (4). 10.1590/0104-07072016005320015.

Santos, T. L. S. S., Silveira, M. B. \& Rezende, H. H. A. (2019). A importancia do exame citopatológico na prevenção do câncer do colo uterino. Enciclopédia Biosfera, Centro Científico Conhecer - Goiânia, 16 (29). 10.18677/EnciBio_2019A151.

Souza, G. D. S., Oliveira, R. A. A., Stevanin, A., Sousa, M. F. \& Almeida, E. C. (2013). A concepção das mulheres de Mirandópolis-São Paulo acerca do exame de Papanicolau. Revista de Enfermagem da UFSM, 3 (3). 10.5902/217976929647.

World Health Organization (WHO) (2002). National cancer control programmes: policies and managerial guidelines. (2a ed.), WHO. https://www.who.int/en/. 